\title{
Analysis of biochemical divergence for classification of six native silkworm based on total cholesterol, triglycerides, high density lipoproteins (HDL) cholesterol, low density lipoproteins (LDL) cholesterol, total lipase and lipids
}

\author{
Alireza Seidavi \\ Department of Animal Science, Rasht Branch, Islamic Azad University, Rasht, Iran. \\ E-mail: alirezaseidavi@iaurasht.ac.ir. \\ Accepted 30 July, 2011
}

\begin{abstract}
This research studies a comparison between the levels and the abundance total cholesterol, triglycerides, HDL cholesterol (High Density Lipoproteins), LDL cholesterol (Low Density Lipoproteins), total lipase and lipids in six native groups of silkworm. These six biochemical parameters and enzymes play important roles in the silk production, digestion and other physiological activities. Other aim of this experiment was study and classification of these six native silkworm based on biochemical parameters. In 5th day of 5th instar, heamolymph sampled using standard method. Sampled heamolymph transferred to laboratory. Heamolymph was obtained by cutting abdominal proleg and collected into $1.5 \mathrm{ml}$ tube containing a few granules of phenylthiourea to prevent melanization. After 10 min centrifugation at $10000 \mathrm{rpm}$, the supernatant was used. Pellets was discarded also. The supernatant was transferred to new tubes and was preserved at $-20^{\circ} \mathrm{C}$ until the onset of the experiments. Hierarchical agglomerative clustering was done by using NTSYS-pc, based on Un weighted pair-group method using arithmetic average (UPGMA). From obtained results, it is showed that amount of total lipase in six studied local varieties included between 79.26 to $176.08 \mathrm{IU} / \mathrm{L}$. Among studied local varieties, the highest level of total lipase belonged to Pink Khorasan (176.08 IU/L), and Lemon Haratee and Baghdadi variety $(79.26 \mathrm{IU} / \mathrm{L})$ remained at lower level than other varieties. Other varieties were between these two groups. Meanwhile statistical differences between studied varieties for this trait were not significant $(P>0.05)$. Analyzed varieties were divided into three distinct groups. At cross 2.53, two clusters were formed which classified into subgroups in crosses of 0.80 . Frequent divisions were also observed in major groups. First group includes Baghdadi variety and second group includes Pink Khorasan variety.
\end{abstract}

Key words: Silkworm, triglyceride, hierarchical agglomerative cluster, biochemical marker.

\section{INTRODUCTION}

Silkworm has been used by people for over 4,000 years to make silk. The practice first began in China in about 2600 BC. The Chinese kept the secret of producing silk

Abbreviations: HDL, High density lipoproteins; LDL, low density lipoproteins; ISRC, Iran silkworm research center; ANOVA, analysis of variance; UPGMA, unweighted pair-group method using arithmetic average. for thousands of years, trading silk to Europe and the Middle East. But eventually the secret of how to get silk from the silkworm was learned by other countries. More recently it has learned how to make silk-like material from synthetic materials, but the demand for real silk is still high, and silkworms are still raised for the silk threads they produce. Indigenous peoples are ethnic groups that are defined as indigenous according to one of the various definitions of the term, there is no universally accepted definition, but most of which carry connotations of being 
the original inhabitants of a territory. There are six local silkworm races in Iran Silkworm Research Center (ISRC) and there are not any data regarding some of their enzyme and biochemical markers. On the other hand, there are positive correlations between some biochemical markers and production characteristics in silkworm. Hence, this research studies a comparison between the levels and the abundance total cholesterol, triglycerides, HDL cholesterol LDL cholesterol total lipase and lipids in six native groups of silkworm. These six biochemical parameters and enzymes play important roles in the silk production, digestion and other physiological activities. Other aim of this experiment was study and classification of these six native silkworm based on biochemical parameters.

\section{MATERIALS AND METHODS}

This study was conducted in ISRC and Islamic Azad University, Rasht Branch, Iran during 2010. Geographic location and climate of research conducted data used in this study were collected from the ISRC which is the original trustee in sericulture researches in Iran. This center is located in Rasht district Pasikhan. This region has a mean temperature of $17^{\circ} \mathrm{C}$, annual rainfall $1450 \mathrm{~mm}$, latitude and longitude $49^{\circ} 36^{\prime}$ and $34^{\circ} 16^{\prime}$ and -6.9 altitudes from sea level. The materials for this experiment were six local silkworm varieties which were conserved and produced by ISRC. It was applied favorite conditions for moth emergence such as $25^{\circ} \mathrm{C}$ and $75 \%$ relative humidity in hatching room for 12 days. Local varieties were reared under standards protocols in all rearing steps. Silkworm eggs were hatched and brushed. Disease-free eggs of the local silkworm varieties were used. In order to make proper coordination in embryos growth, silkworm egg to embryos rotation stage (day 6 to 7) exposed to natural daylight and darkness at night and after being up at this stage to change the color stage of the egg they have exposure $18 \mathrm{~h}$ light and $6 \mathrm{~h}$ darkness. Then which completion of changing the color of the egg (more than 90\%), complete darkness for three days given and on the morning of the day fourteen with the supply of light, the eggs were hatching. Silkworm rearing techniques of humidity, temperature, light, young and mature silkworm rearing were conducted following the standard procedure of ESCAP (1993). Scientific technology of silkworm rearing was followed according to standard method. Rearing in young silkworm period was performed by chopped leaves and paraffin paper coverage and in the adult period it was performed with leaves and branches.

In 5th day of 5 th instar, heamolymph sampled using standard method. Sampled heamolymph transferred to laboratory. Heamolymph was obtained by cutting abdominal proleg and collected into $1.5 \mathrm{ml}$ tube containing a few granules of phenylthiourea to prevent melanization. After $10 \mathrm{~min}$ centrifugation at $10000 \mathrm{rpm}$, the supernatant was used. Pellets were discarded also. The supernatant was transferred to new tubes and was preserved at $-20^{\circ} \mathrm{C}$ until the onset of the experiments. Six local silkworm varieties were used in the present study. These varieties included (1) Lemon Khorasan; (2) Lemon Haratee, (3) White Haratee; (4) Yellow Haratee; (5) Pink Khorasan; and (6) Baghdadi. Data recorded for this study were total cholesterol, triglycerides, HDL cholesterol and LDL cholesterol total lipase and lipids. These parameters were measured using commercial and experimental kits (Thomas, 1998). Furthermore data above $70 \%$ or below $30 \%$, undergone inverse sin transformation $\left(Z=A r c s i n P_{i j}^{1 / 2}\right)$ and data between $0-1$, undergone square transformation $\left(P^{1 / 2}\right)$. The data were subjected to analysis of variance (ANOVA) to determine if the differences found between treatments and the differences between treatments were significant. For analysis of variance, Tukey's studentized range (HSD) test in a complete randomized design was used at $\alpha=0.05$. The grouping methods allowed us to subdivide observations into several subgroups in such a way that we obtained homogeneity inside the subgroups and heterogeneity among the subgroups. Hierarchical agglomerative clustering was done by using NTSYS-pc, version 2.02e (Rohlf, 1998) based on UPGMA approach and the resulting clusters were expressed as dendrograms (Sneath and Sokal, 1973). This method employed for grouping, UPGMA, uses the average distance among all the equal genotypes for the formation of each group (Cruz and Regazzi, 2001; Zanatta et al., 2009). The clustering was based on the squared Euclidean distance. The average linkage between two groups is considered as the average of distance between all pairs of cases with one number from each group. Hierarchical clustering analysis was carried out by considering all studied parameters individually and together.

\section{RESULTS AND DISCUSSION}

Obtained results are summarized in Table 1 and Figures 1 to 7.

\section{Total cholesterol}

From obtained results, it is showed that amount of total cholesterol in six studied local varieties include between 1.00 to $11.53 \mathrm{mg} / \mathrm{dl}$. Among studied local varieties, the highest level of total cholesterol belonged to Yellow Haratee $(11.53 \mathrm{mg} / \mathrm{dl})$, and Baghdadi variety $(1.00 \mathrm{mg} / \mathrm{dl})$ remained at lower level than other varieties. Other varieties were between these two groups. Meanwhile statistical differences between studied varieties for this trait were significant $(P<0.05)$. Figure 1 obtained from hierarchical analysis of these varieties, represents phylogeny classification of six studied varieties based on total cholesterol parameter. On the basis of these dendrograms, analyzed varieties were divided into three distinct groups. At cross 2.01, two clusters were formed which classified into subgroups in crosses of 0.80 and 0.30 . Frequent divisions were also observed in major groups. First group include white haratee and yellow haratee varieties.

\section{Triglycerides}

From obtained results, it is showed that amount of $\mathrm{HDL}$ Cholesterol in six studied local varieties included between 70.73 to $172.07 \mathrm{mg} / \mathrm{dl}$. Among studied local varieties, the highest level of HDL Cholesterol belonged to Pink Khorasan $(172.07 \mathrm{mg} / \mathrm{dl})$, and Lemon Haratee variety $(70.73 \mathrm{mg} / \mathrm{dl})$ remained at lower level than other varieties. Other varieties were between these two groups. Meanwhile statistical differences between studied varieties for this trait were significant $(P<0.05)$. Figure 2 obtained from hierarchical analysis of these varieties, represents phylogeny classification of six studied varieties based on HDL Cholesterol parameter. On the basis of these 
Table 1. Mean comparison ( \pm SEM) of biochemical parameters in heamolymph of six studied local silkworm varieties*.

\begin{tabular}{|c|c|c|c|c|c|c|c|}
\hline \multirow[b]{2}{*}{ Parameter } & \multirow[b]{2}{*}{ Unit } & \multicolumn{6}{|c|}{ Variety } \\
\hline & & $\begin{array}{c}\text { Lemon } \\
\text { khorasan (1) }\end{array}$ & $\begin{array}{c}\text { Lemon } \\
\text { haratee (2) }\end{array}$ & $\begin{array}{c}\text { White } \\
\text { haratee (3) }\end{array}$ & $\begin{array}{c}\text { Yellow } \\
\text { haratee (4) }\end{array}$ & $\begin{array}{c}\text { Pink } \\
\text { khorasan (5) }\end{array}$ & Baghdadi (6) \\
\hline Total cholesterol & $\mathrm{mg} / \mathrm{dl}$ & $2.50 \pm 0.28^{d}$ & $5.01 \pm 0.01^{c}$ & $10.00 \pm 0.57^{b}$ & $11.53 \pm 0.29^{a}$ & $2.00 \pm 0.00^{d}$ & $1.00 \pm 0.00^{\mathrm{e}}$ \\
\hline Triglycerides & $\mathrm{mg} / \mathrm{dl}$ & $87.79 \pm 0.41^{\mathrm{c}}$ & $70.73 \pm 0.89^{e}$ & $115.88 \pm 1.49^{b}$ & $113.87 \pm 0.47^{b}$ & $172.07 \pm 1.03^{\mathrm{a}}$ & $77.25 \pm 0.25^{d}$ \\
\hline HDL cholesterol (high density lipoproteins) & $\mathrm{mg} / \mathrm{dl}$ & $1.00 \pm 0.00^{c}$ & $2.50 \pm 0.28^{b}$ & $6.02 \pm 0.02^{a}$ & $1.00 \pm 0.00^{\mathrm{C}}$ & $1.00 \pm 0.00^{\mathrm{c}}$ & $1.00 \pm 0.00^{\mathrm{c}}$ \\
\hline LDL cholesterol (low density lipoproteins) & $\mathrm{mg} / \mathrm{dl}$ & $91.08 \pm 0.91^{\mathrm{c}}$ & $1.00 \pm 0.00^{c}$ & $2.50 \pm 0.28^{b}$ & $7.02 \pm 0.23^{\mathrm{a}}$ & $1.00 \pm 0.00^{c}$ & $0.00 \pm 0.00^{\mathrm{e}}$ \\
\hline Total lipase & IU/L & $91.08 \pm 0.91^{\mathrm{C}}$ & $79.26 \pm 0.63^{d}$ & $134.44 \pm 0.72^{b}$ & $134.44 \pm 0.72^{\mathrm{b}}$ & $176.08 \pm 1.06^{\mathrm{a}}$ & $79.26 \pm 0.26^{d}$ \\
\hline Lipids & $\mathrm{mg} / \mathrm{dl}$ & $10.03 \pm 0.03^{c}$ & $11.53 \pm 0.29^{c}$ & $12.54 \pm 0.86^{\mathrm{ab}}$ & $10.53 \pm 0.86^{c}$ & $15.55 \pm 1.44^{b}$ & $25.58 \pm 2.02^{a}$ \\
\hline
\end{tabular}

*Means in each row followed by the same letters are not significantly different at $\alpha=0.05$.
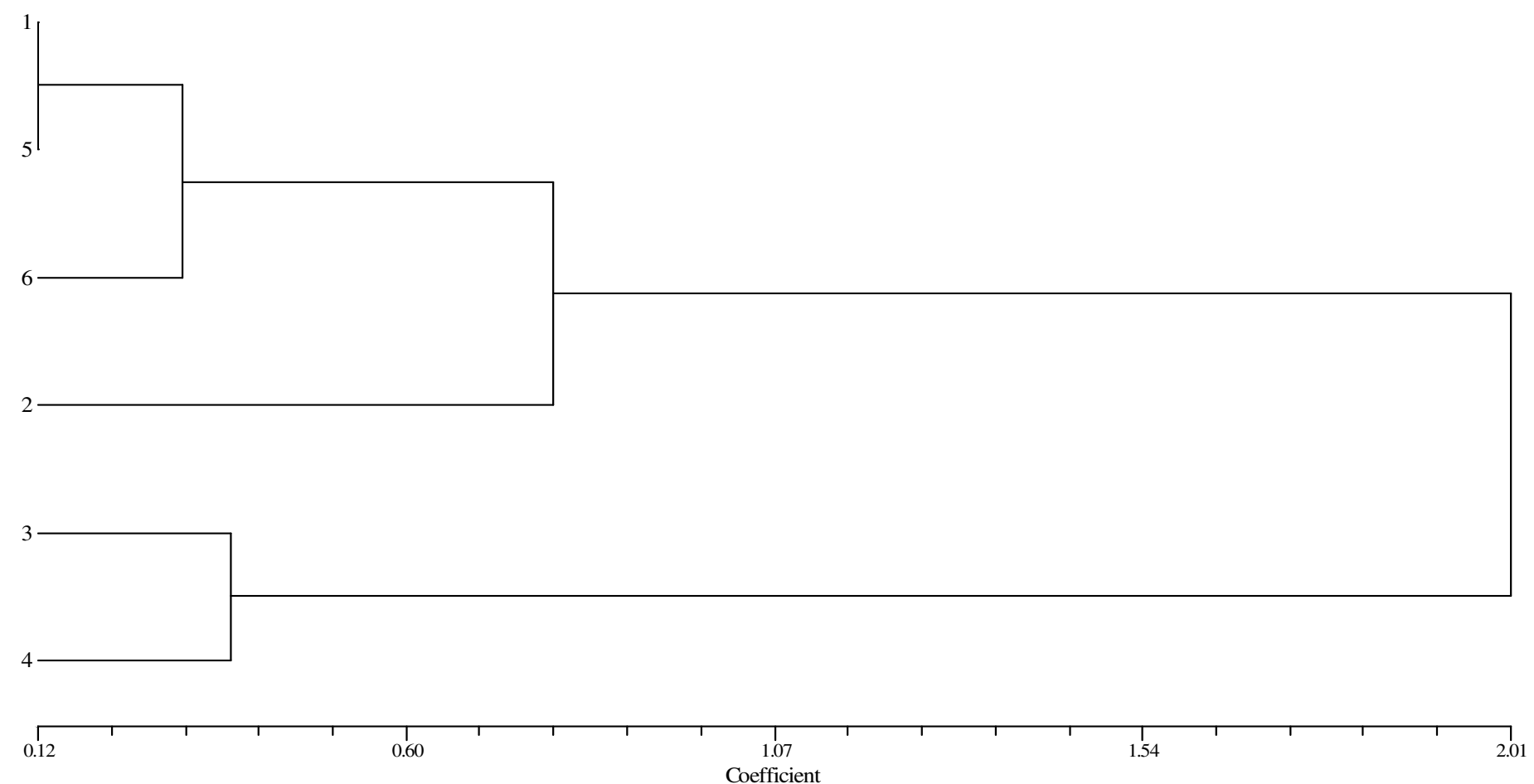

Figure 1. Cluster analysis of 6 local studied silkworm varieties based on heamolymph total cholesterol .

(1) Khorasan lemon (2) lemon haratee (3) white haratee (4) yellow haratee (5) pink khorasan (6) Baghdadi. 


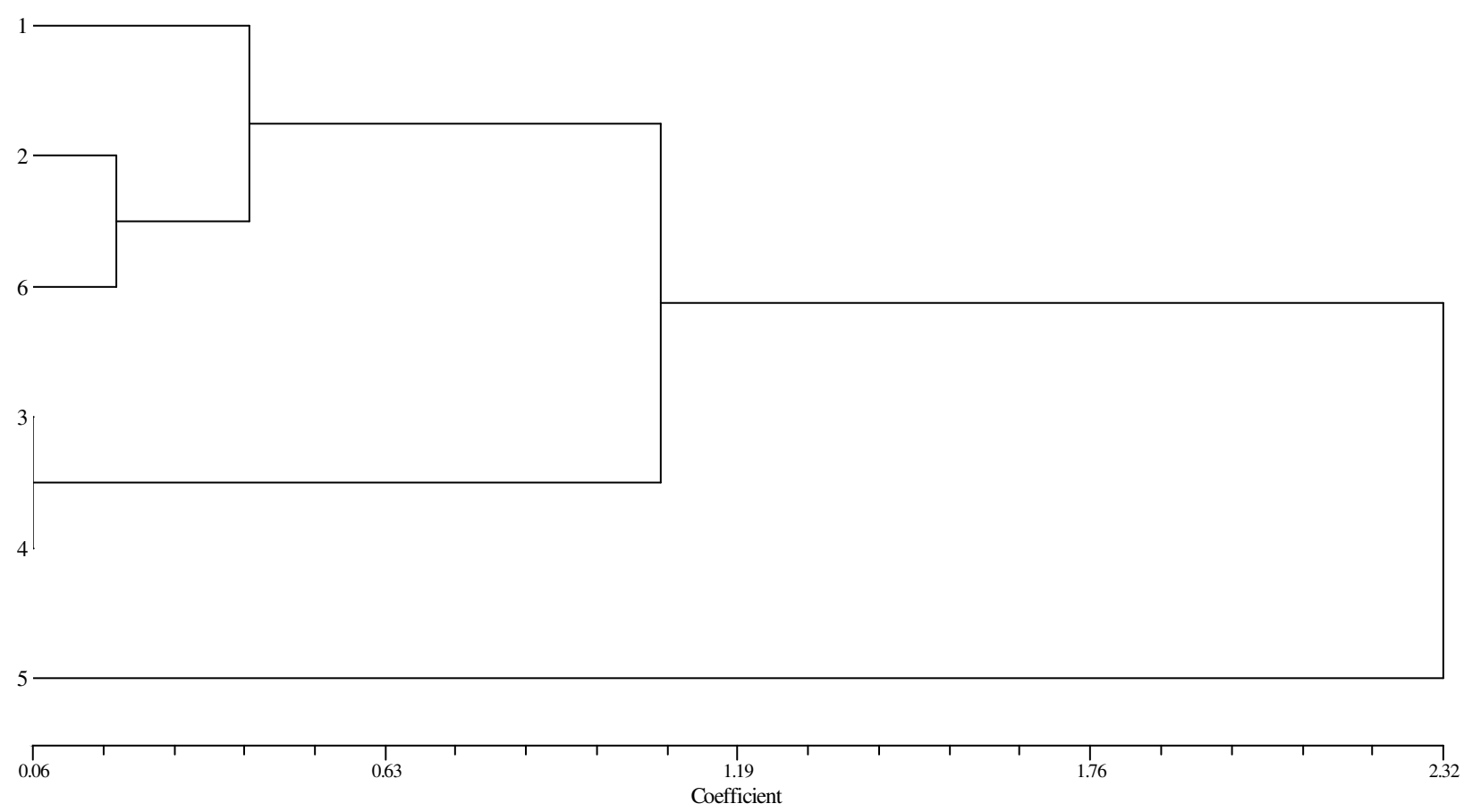

Figure 2. Cluster analysis of 6 local studied silkworm varieties based on heamolymph triglycerides.

(1) Khorasan lemon (2) Lemon haratee (3) White haratee (4) Yellow haratee (5) Pink khorasan (6) Baghdadi.

dendrograms, analyzed varieties were divided into three distinct groups. At cross 2.33, two clusters were formed which classified into subgroups in crosses of 1.00 . Frequent divisions were also observed in major groups. First group include pink khorasan variety. Second group included other varieties.

\section{HDL cholesterol (high density lipoproteins)}

From obtained results, it is showed that amount of HDL Cholesterol in six studied local varieties included between 1.00 and $6.02 \mathrm{mg} / \mathrm{dl}$. Among studied local varieties, the highest level of $\mathrm{HDL}$ Cholesterol belonged to white haratee $(6.02 \mathrm{mg} / \mathrm{dl})$, and most of other varieties (1.00 $\mathrm{mg} / \mathrm{dl}$ ) remained at lower level than other varieties. Meanwhile statistical differences between studied varieties for this trait were significant $(P<0.05)$. Figure 3 obtained from hierarchical analysis of these varieties, represents phylogeny classification of six studied varieties based on HDL Cholesterol parameter. On the basis of these dendrograms, analyzed varieties were divided into three distinct groups. At cross 2.56, two clusters were formed which classified into subgroups in crosses of 0.80 . Frequent divisions were also observed in major groups. First group included white haratee variety. Second group included other varieties.

\section{LDL cholesterol (low density lipoproteins)}

From obtained results, it is showed that amount of LDL cholesterol in six studied local varieties included between 0.00-7.02 $\mathrm{mg} / \mathrm{dl}$. Among studied local varieties, the highest level of LDL cholesterol belonged to yellow haratee $(7.02 \mathrm{mg} / \mathrm{dl})$, and Baghdadi variety $(0.00 \mathrm{mg} / \mathrm{dl})$ remained at lower level than other varieties. Other varieties were between these two groups. Meanwhile statistical differences between studied varieties for this trait were significant $(P<0.05)$. Figure 4 obtained from hierarchical analysis of these varieties, represents phylogeny classification of six studied varieties based on LDL cholesterol parameter. On the basis of these dendrograms, analyzed varieties were divided into four distinct groups. At cross 2.54, two clusters were formed which classified into subgroups in crosses of 0.77 and 0.30 Frequent divisions were also observed in major groups. First group include yellow haratee variety. Second group include other varieties.

\section{Total lipase}

From obtained results, it is showed that amount of total lipase in six studied local varieties included between $79.26-176.08 \mathrm{IU} / \mathrm{L}$. Among studied local varieties, the highest level of total lipase belonged to Pink Khorasan (176.08 IU/L), and lemon haratee and Baghdadi variety $(79.26 \mathrm{IU} / \mathrm{L})$ remained at lower level than other varieties. Other varieties were between these two groups. Meanwhile statistical differences between studied varieties for this trait were not significant $(P>0.05)$. Figure 5 obtained from hierarchical analysis of these varieties, 


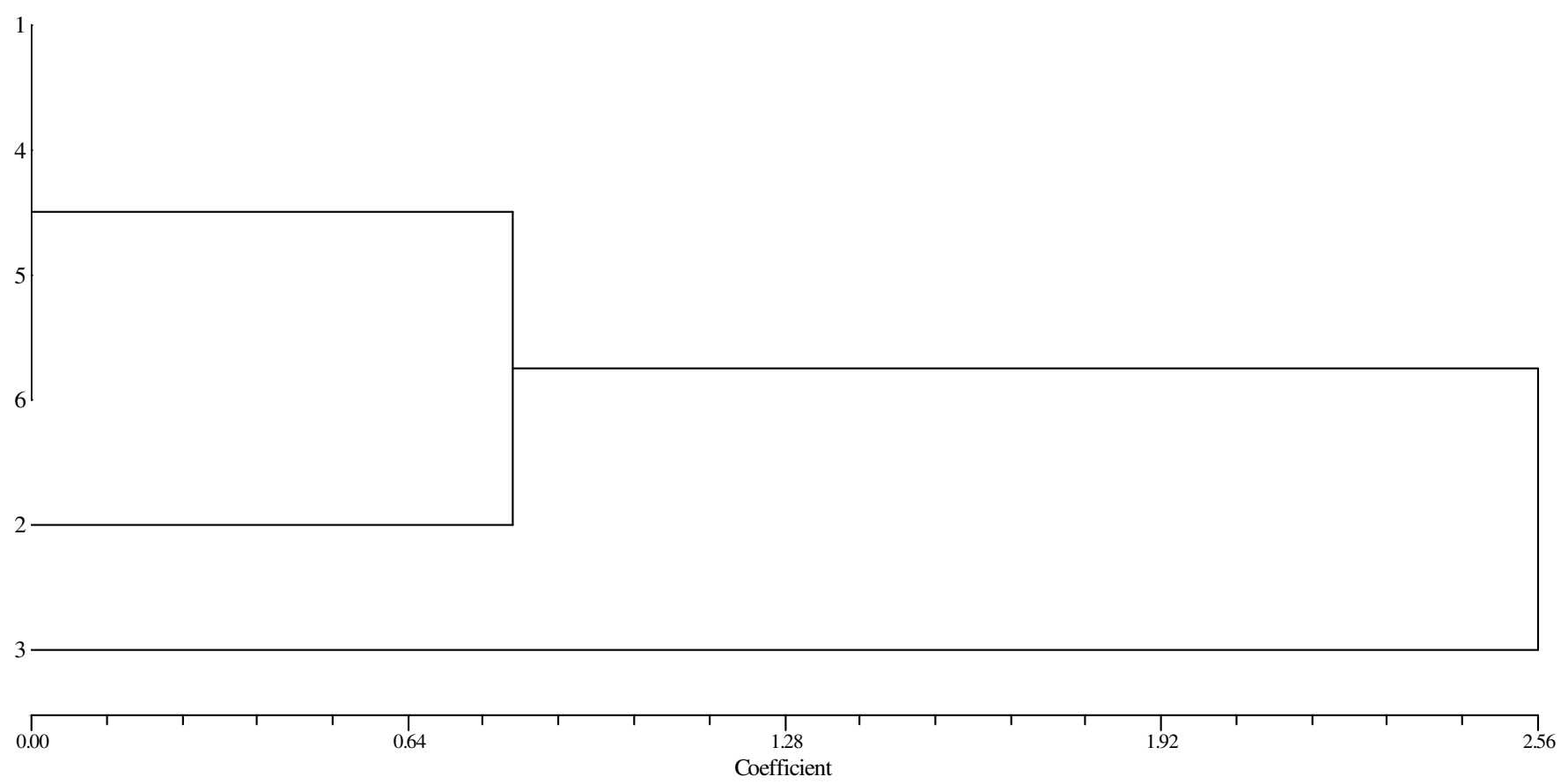

Figure 3. Cluster analysis of 6 local studied silkworm varieties based on heamolymph HDL cholesterol (high density lipoproteins). (1) Khorasan lemon (2) Lemon haratee (3) White haratee (4) Yellow haratee (5) Pink khorasan (6) Baghdadi.

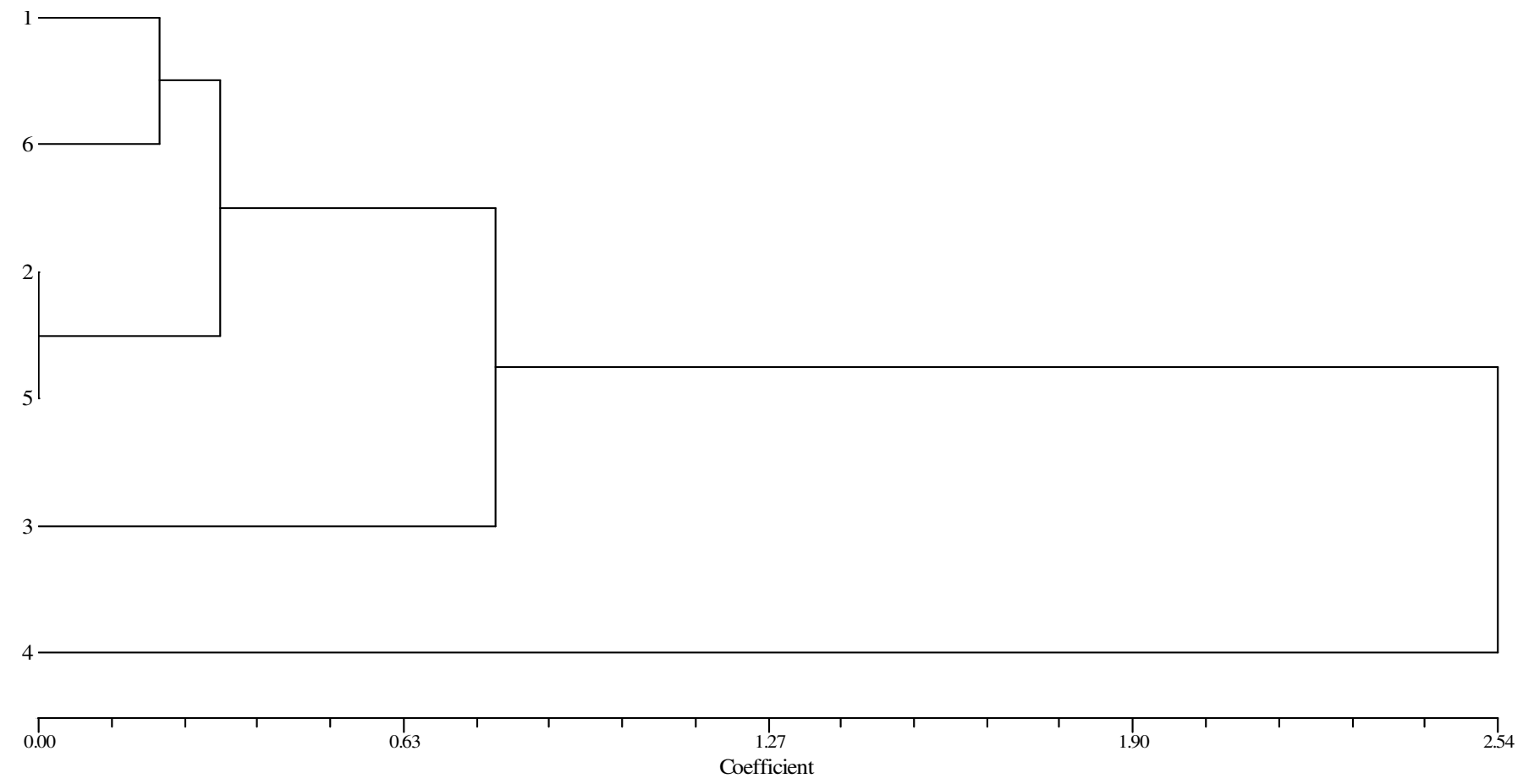

Figure 4. Cluster analysis of 6 local studied silkworm varieties based on heamolymph LDL cholesterol (low density lipoproteins). (1) Khorasan lemon (2) lemon haratee (3) white haratee (4) yellow haratee (5) pink khorasan (6) Baghdadi.

represents phylogeny classification of six studied varieties based on total lipase parameter. On the basis of these dendrograms, analyzed varieties were divided into three distinct groups. At cross 2.53, two clusters were 


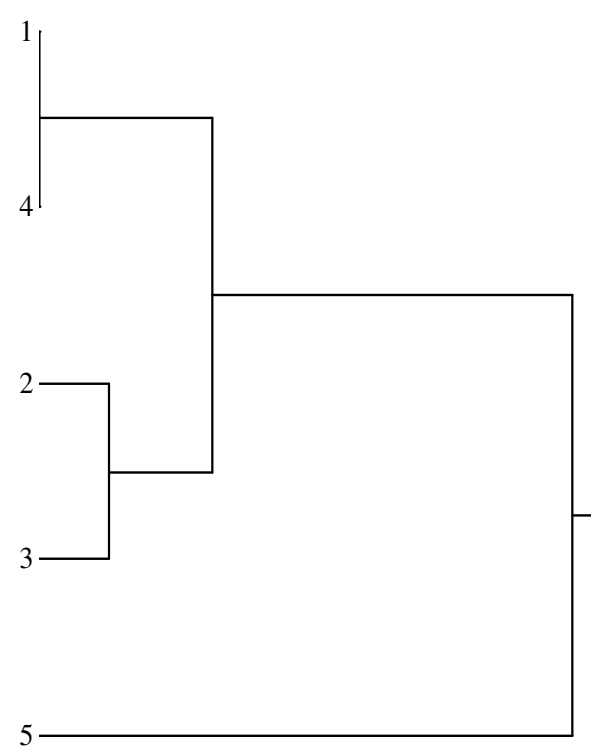

6

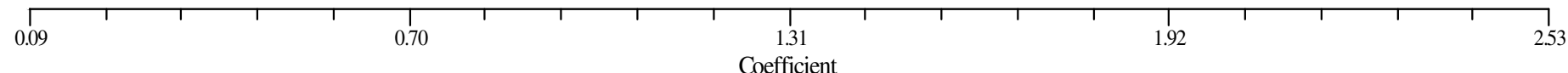

Figure 5. Cluster analysis of 6 local studied silkworm varieties based on heamolymph total lipase.

(1) Khorasan lemon (2) lemon haratee (3) white haratee (4) yellow haratee (5) pink khorasan (6) Baghdadi.

formed which classified into subgroups in crosses of 0.80 . Frequent divisions were also observed in major groups. First group included Baghdadi variety and second group included Pink Khorasan variety.

\section{Lipids}

From obtained results, it is showed that amount of lipids in six studied local varieties include between 10.53-25.58 $\mathrm{mg} / \mathrm{dl}$. Among studied local varieties, the highest level of lipids belonged to Baghdadi $(25.58 \mathrm{mg} / \mathrm{dl})$, and white haratee variety $(10.53 \mathrm{mg} / \mathrm{dl})$ remained at lower level than other varieties. Other varieties were between these two groups. Meanwhile statistical differences between studied varieties for this trait were significant $(P<0.05)$. Figure 6 obtained from hierarchical analysis of these varieties, represents phylogeny classification of six studied varieties based on lipids parameter. On the basis of these dendrograms, analyzed varieties were divided into three distinct groups. At cross 1.82, two clusters were formed which classified into subgroups in crosses of 1.20. Frequent divisions were also observed in major groups. First group included lemon khorasani, lemon haratee and Baghdadi varieties.

\section{Total cholesterol, triglycerides, HDL cholesterol, LDL cholesterol, total lipase and lipids}

Figure 7 obtained from hierarchical analysis of these varieties, represents phylogeny classification of six studied varieties based on total Cholesterol, triglycerides, HDL cholesterol, LDL cholesterol, total lipase and lipids parameters. On the basis of these dendrograms, analyzed varieties were divided into four distinct groups. At cross 4.40, two clusters were formed which classified into subgroups in crosses of 4.00 and 3.00 . Frequent divisions were also observed in major groups. First group included lemon khorasan, lemon haratee and Baghdadi. The cluster analysis provides scope for adopting a recombinational breeding program using distant cluster members. Thus, the sub grouping of high-yielding bivoltine strains offers an opportunity to exploit the genetically differences between high-yielding strains. The clustering also indicates the possibility for recombining low and high-yielding members from genetically distant clusters.

The results presented here establish its usefulness in realizing a better projection of the genetical difference between silkworm strains of different yield potentials (Chatterjee and Data, 1992; Salehi Nezhad et al., 2009). 


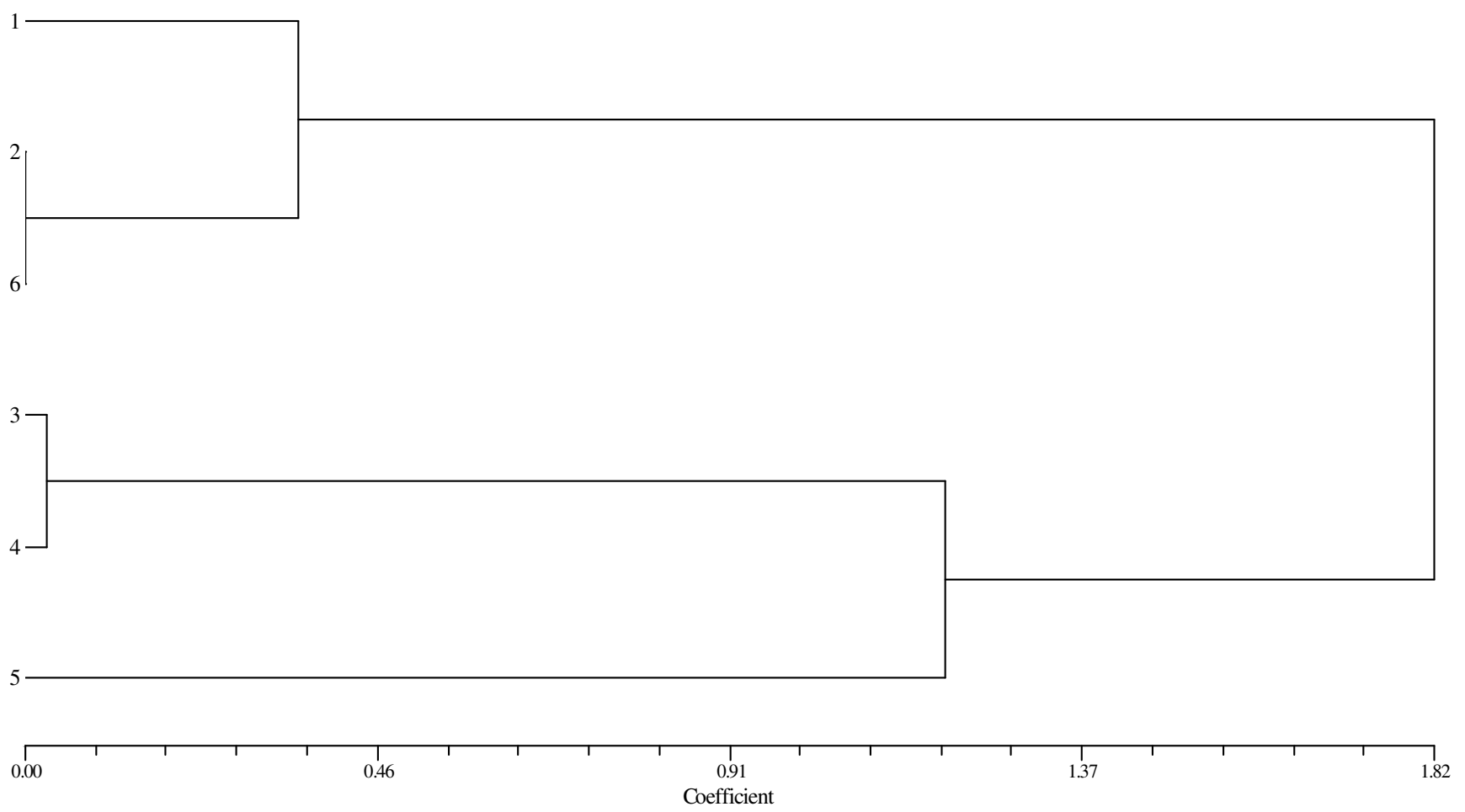

Figure 6. Cluster analysis of 6 local studied silkworm varieties based on heamolymph total lipids.

(1) Khorasan lemon (2) Lemon haratee (3) White haratee (4) Yellow haratee (5) Pink khorasan (6) Baghdadi.
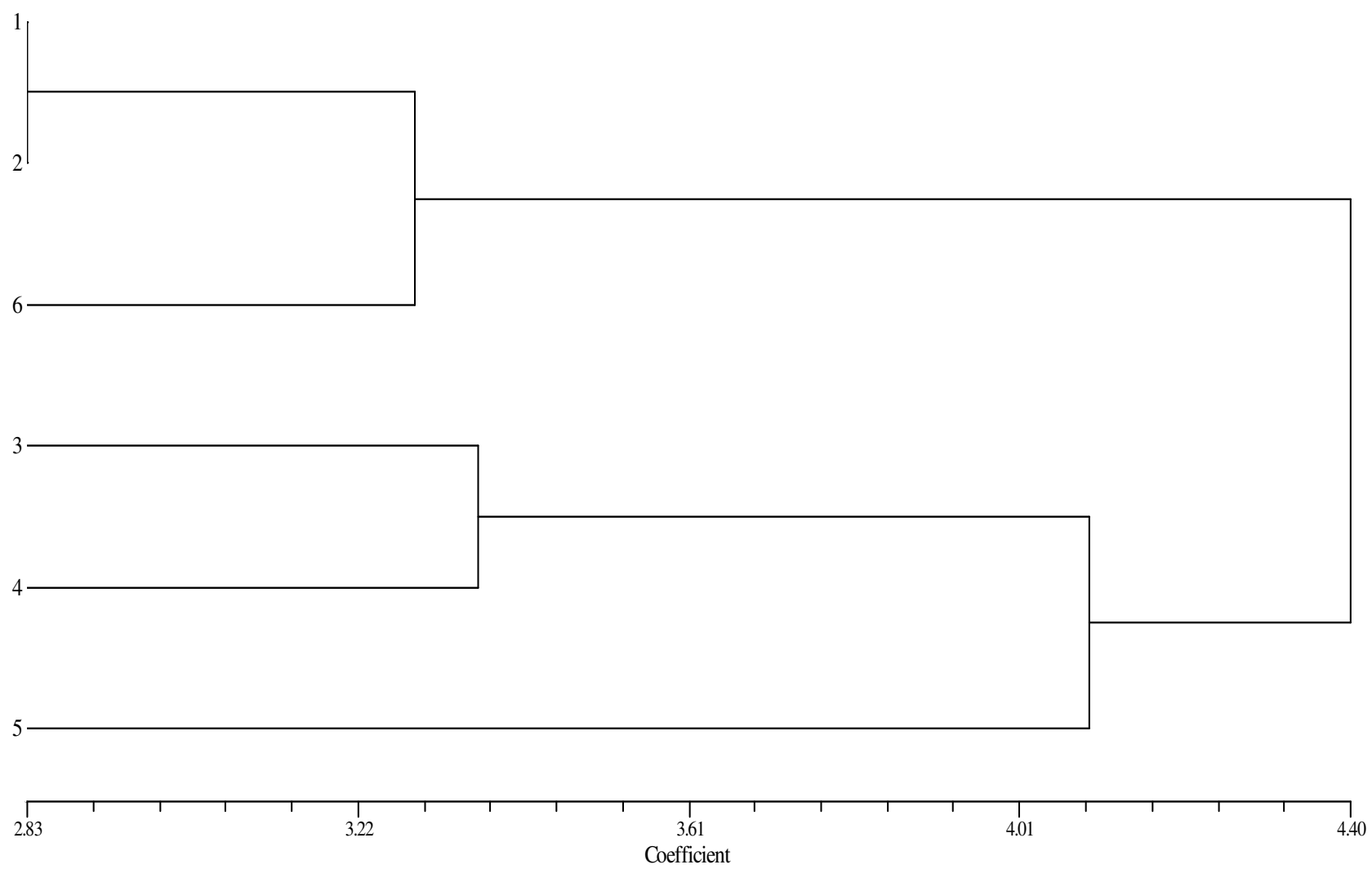

Figure 7. Cluster analysis of 6 local studied silkworm varieties based on all studied heamolymph biochemical parameters. (1) Khorasan lemon (2) Lemon haratee (3) White haratee (4) Yellow haratee (5) Pink khorasan (6) Baghdadi. 
Researchers emphasized that the high genetic variation might not give always a high genetic diversity in the inbreeding population of same species. This further confirmed the earlier report that the genetic diversity is not always related with geographical diversity (Ramamohana and Nakada, 1998). It is obvious that the silkworm germplasm contributes the potential raw materials for breeding having wide genetic variation in their genotypic expression besides additive effect due to inbreeding (Kumaresan et al., 2007; Salehi Nezhad et al., 2009). Hierarchical agglomerative clustering was done based on complet, single, UPGMA, UPGMC, FLEXI, WARD and average approaches. All these approaches yielded optimal some cluster solution after using Ward's cluster algorithm, there was a notable discrepancy in the size and shape of the clusters. Nevertheless, as Knezovi et al. (2005) stated evaluation of the results using criteria proposed by Franco et al. (1998) showed that all methods have similar efficiency, on the basis of number of influential variables criteria (Salehi Nezhad et al., 2010). As Salehi Nezhad et al. (2010) stated Bombyx mori strains have been reared in different regions of the world and different strains have evolved because of changes in their phenotype and genotype over time (Mirhosseini et al., 2007). Based on one hypothesis, all the strains during a long period have been differentiated from a monovoltine Chinese variety (Chatterjee and Data, 1992; Mirhosseini et al., 2007).

\section{ACKNOWLEDGMENTS}

This experiment was supported by the Islamic Azad University, Rasht Branch, Iran. The author sincerely thanks anonymous reviewers for comments on earlier drafts of this manuscript. The author also acknowledges the kind advice of Mr. Mavvajpour, Mr. Bizhannia, Mrs. KH. Taieb Naeemi, Mr. Y. Kheirkhah and Mr. M. Salehi Nezhad for valuable comments and helpful assistances.

\section{REFERENCES}

Chatterjee SN, Datta RK (1992). Genetics Laboratory, Central Sericultural Research and Training Institute, Mysore-570008, Karnataka, India.

Cruz CD, Regazzi AJ (2001). Modelos biométricos aplicados ao melhoramento genético. 2nd ed, Imprensa Universitária, 390 p.

ESCAP (1993). Principle and Techniques of Silkworm Breeding. United Nations, New York.

Franco J, Crossa J, Villasenor J, Taba S, Eberhart SA (1998). Classifying genetic resources by categorical and continuous variables. Crop Sci., 38: 1688-1696.

Knezovi Z, Gunjaca J, Satovic Z, Kolak I (2005). Comparison of Different Methods for Classification of Gene Bank Accessions. Agric. Conspectus Sci., 70(3): 87-91.

Kumaresan P, Sinha RK, Raje Urs S (2007). An analysis of genetic variation and divergence in Indian tropical polyvoltine silkworm (Bombyx mori L.) genotypes. Caspian J. Enviiron. Sci., 5(1): 11-17.

Mirhosseini SZ, Dalirsefat SB, Pourkheirandish M (2007). Genetic Characterization of Iranian Native Bombyx mori Strains Using Amplified Fragment Length Polymorphism Markers. J. Econ. Entomol., 100(3): 939-945.

Ramamohana Rao P, Nakada T (1998). Clustering of polyvoltine strains of the silkworm $B$. mori by image processing method: Significance of cocoon size and weight variables. Indian J. Sericol., 37: 33-39.

Rohlf FJ (1998). NTSYSpc: numerical taxonomy and multivariate system, version 2.02e. Exeter Software, New York.

Salehi Nezhad M, Mirhosseini SZ, Gharahveysi S, Mavvajpour M, Seidavi AR (2009). Analysis of Genetic Divergence for Classification of Morphological and Larval Gain Characteristics of Peanut Cocoon Silkworm (Bombyx mori L.) Germplasm. Am-Eurasian J. Agric. Environ. Sci., 6(5): 600-608.

Salehi Nezhad M, Mirhosseini SZ, Gharahveysi S, Mavvajpour M, Seidavi AR (2010). Investigation on intera-specific biodiversity of 51 peanut cocoon strains of Iran silkworm (Bombyx mori) germplasm based on reproductive traits. Biotechnology, 9(2): 149-156.

Sneath PHA, Sokal RR (1973). Numerical taxonomy. Freeman, San Francisco, CA.

Thomas L (1998). Clinical Laboratory Diagnostics. $1^{\text {st }}$ ed. Frankfurt THBooks verlagsgesellschaft.

Zanatta DB, Bravo JP, Barbosa JF, Munhoz REF, Fernandez MA (2009). Evaluation of Economically Important Traits from Sixteen Parental Strains of the Silkworm Bombyx mori L (Lepidoptera: Bombycidae). Neotrop. Entomol., 38(3): 327-331. 